\title{
Seeking coherence and switching reasoning after forgetting an equation
}

\author{
Katherine Gifford \\ Department of Physics, Adelphi University, 1 South Ave, Garden City, NY, 11530
}

Gabriel S. Ehrlich

Departments of Physics and Curriculum \& Instruction, University of Illinois Urbana-Champaign, 1110 W Green St, Urbana, IL, 61801

Engin Bumbacher

Haute École Pédagogique Vaud, Avenue de Cour 33,1014, Lausanne, Switzerland

Eric Kuo

Departments of Physics and Curriculum \& Instruction, University of Illinois Urbana-Champaign, 1110 W Green St, Urbana, IL, 61801

\begin{abstract}
Selection and application of an appropriate equation is a key step in instructional problem-solving strategies. But what if one cannot exactly recall the relevant equation? We are currently investigating how coherence between physical and mathematical reasoning opens multiple solution pathways for qualitative physics problems. In one-on-one interviews, community college students, 4-year college undergraduates, and graduate students solved qualitative introductory-level E\&M questions. Analysis of 18 interviews showed that while many students had initial difficulty in recalling a relevant equation, they demonstrated strategies for making progress. We present two cases from the data corpus. One interviewee was unsure whether the time constant for an RC circuit is RC or $1 /(\mathrm{RC})$ and sought coherence with qualitative reasoning to determine the correct expression. Another student switched to reasoning qualitatively from a physical model after noting that they could not recall a relevant equation. These examples show how mathematical and physical reasoning is opportunistically incorporated in students' problem solving.
\end{abstract}

\footnotetext{
2021 PERC Proceedings edited by Bennett, Frank, and Vieyra; Peer-reviewed, doi.org/10.1119/perc.2021.pr.Gifford

Published by the American Association of Physics Teachers under a Creative Commons Attribution 4.0 license.

Further distribution must maintain the cover page and attribution to the article's authors.
} 


\section{INTRODUCTION}

On the Maryland Physics Expectations Survey (MPEX2), students are asked to rate on a 5-point Likert scale to what extent they agree with the following statement: "If I don't remember a particular equation needed for a problem in an exam, I can probably figure out an (ethical!) way to come up with it, given enough time" [1]. The favorable response to this question is "agree," reflecting an expert view that one can (re)construct physics knowledge. While this question uncovers students' beliefs about whether or not they can make progress while problem-solving even if they forget a relevant equation, it does not clarify exactly what strategies students can use to make that progress. This paper presents two cases of how students make progress on physics problems when they cannot remember a relevant equation. Incorporating the teaching of such productive reasoning strategies could potentially benefit student problem solving and epistemological beliefs.

The selection and application of relevant equations are explicit steps in PER-based conceptualizations of quantitative problem solving [2-6]. And while the use of equations in qualitative problem solving is less documented, Park demonstrates that students can utilize equations to explore a given physical situation while answering qualitative questions [7]. Although these frameworks describe a successful problem-solving pathway, they do not suggest corrective strategies when a roadblock appears, such as when a key equation is forgotten.

One question investigated in this paper is: how can students reconstruct (partially) forgotten equations? One possible corrective strategy is that the equation could be rederived. For instance, the time constant for RC circuits $(\tau=R C)$ can be derived by applying Kirchoff's laws to an $\mathrm{RC}$ circuit and solving a differential equation. Another possible strategy is that students may use their understanding of the physical situation to generate a sensible equation. Sherin [8] found that third-semester physics students were able to directly generate equations that matched their conceptual understanding of physical situations. For instance, to describe the acceleration of a falling object experiencing air resistance, students directly wrote equations of the form $a=\frac{F_{\text {down }}}{m}-\frac{F_{u p}}{m}$ to represent the structure of two opposing influences. Such reasoning that leverages the coherence between qualitative physics understanding and mathematical equations has been investigated under the label of mathematical sensemaking [9-13]. Mathematical sensemaking research models the messy ways that student problem solving deviates from idealized problem-solving procedures, such as understanding and fixing mistakes [9] or constructing solutions from multiple physical, graphical, and symbolic elements [13]. Building on this research, we seek empirical examples of how physics students use mathematical sensemaking to make progress when they forget an equation.
A second question investigated in this paper is: what alternative solutions can students who forget a relevant equation apply that don't explicitly require an equation? While equations are difficult to avoid on quantitative problems, qualitative problems are more likely to be solvable without equations. Through this research project, we also seek empirical examples of how physics students who initially note that they forget a relevant equation turn to other strategies on qualitative physics problems.

In the next sections, we present an overview of the data corpus of interviews, detail one focal problem (the Four Circuits problem) and highlight two cases of how students make progress after forgetting a relevant equation. One student, Gabi, uses their physical understanding of the problem situation to reconstruct the equation by deciding between two likely candidates for the equation. Sam uses a similar physical understanding of the problem situation to instead answer the question qualitatively. These two cases provide empirical examples of how students adapt when forgetting a relevant equation on a physics problem.

\section{METHODS}

The data corpus consists of 18 semi-structured clinical interviews. Participants were students at a community college or students at an elite, private university. The students ranged from undergraduates in introductory E\&M to graduate students. This paper will focus on Sam, a community college student currently enrolled in an introductory course, and Gabi, a rising junior physics major at the elite private university.

In these hour-long interviews, students thought aloud as they answered qualitative, introductory-level E\&M questions. The interviewer maintained a neutral approach, and interjections and follow-up questions were mainly for clarification about statements made by the interviewee. Our analysis is informed by the methodological perspective that reasoning displayed in these interviews are shaped by interviewees' framing of the interviews as well as their interactions with the interviewer $[14,15]$.

Content $\operatorname{logs}$ were created with attention placed on the interviewees reasoning as well as interview dynamics. We attended to the types of physical and mathematical reasoning students used to answer the interview questions. With this focus, two questions from the interview protocol, both involving capacitors, emerged as particularly interesting eliciting rich reasoning from interviewees. As we discussed these episodes and refined our interpretation, codes were inductively developed to categorize common, intriguing features of interviewees' reasoning on these problems, such as "forgot an equation/expression," "searching for coherence," and "anxious/unsure." 
The light bulbs, batteries and wires are all the same in the four circuits. The capacitors in circuits A, B, and C are the same. The gap in capacitor in circuit D is half of the other capacitors. All capacitors have individually been charged by connecting them to a $9 \mathrm{~V}$ battery separately from each other. They are almost fully charged, i.e. they have the maximal possible charge on either plate.

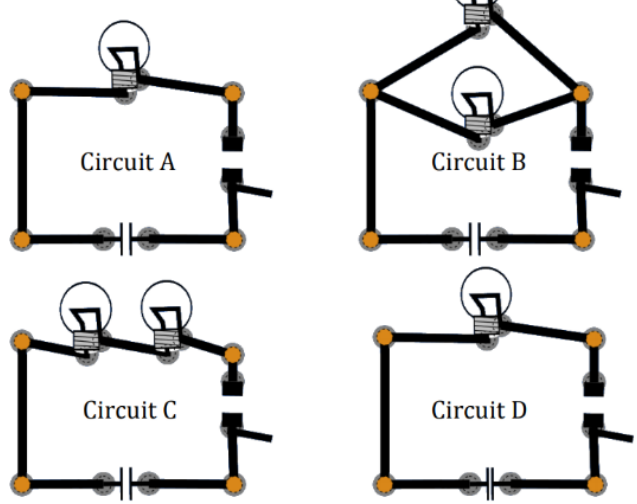

You close the switches in all four circuits at the same time. Each capacitor takes at least 2 seconds to fully discharge. After 1 second, you open all the switches again.

Which capacitor has the most amount of charge left on its plates? Which capacitor has the least amount of charge left on its plates? Explain your reasoning.**

FIG. 1. The "Four Circuits" problem. The correct answer is: Circuit D has the most charge left on its plates. Circuit B has the least amount of charge left.

Across the 18 interviews, mentioning that one forgot or could not remember a relevant equation or mathematical expression, either in part or whole, occurred twelve times by eleven interviewees throughout the two focal questions (noting that five interviewees only received one of these focal questions). This paper presents two cases of what students do when they forget a relevant equation/expression on the "Four Circuits" problem (Fig. 1). The first student, Gabi, used qualitative relationships to determine the proper expression. Despite there being other students who used this approach, Gabi was selected for this case study as their reasoning was particularly concise. Sam, the second student we show, was the only to apply physical analogies and qualitative reasoning to bypass the need for equations. While uncommon in our data, this process showcases an alternative, creative strategy.

We perceived Gabi and Sam as women, Sam as white, and were unsure of Gabi's race. We recognize these perceptions may impact our analysis. As we did not ask participants to answer demographic questions, and to avoid misgendering Gabi or Sam, we use they/them pronouns in our descriptions.

\section{TWO CASES OF FORGETTING AN EQUATION}

\section{A. Gabi determines the correct equation through qualitative reasoning}

After being presented with the Four Circuits problem, Gabi, a rising junior physics major, begins to respond:
32 Gabi: Okay, so. (4s pause) The, I think that there's like, in RC circuits like the time constant of how (5s pause), (one hand partially covers face as the other holds pen) but like the time constant of how long the capacitors take to discharge is

33 Interviewer: Mhm.

34 G: $R C$ ?

35 I: Mhm.

36 G: It's either $R C$ or $1 / R C$ and let's see intuitively which one it should be.

Gabi recognizes that the time constant will be a useful way to determine the solution to the presented problem, but their memory of the time constant expression here is incomplete. With two plausible possibilities in mind, $R C$ or $1 / R C$, Gabi states that they will "intuitively" see which expression it will be (turn 36), though it is ambiguous what type of approach they are imagining. One formal mathematical approach would be to derive the time constant by writing Kirchhoff's voltage law for an $R C$ circuit and solving a differential equation. However, Gabi's subsequent reasoning indicates a different approach in mind, which depends on matching the physical circuit behavior to the mathematical form of the time constant. Gabi continues:

36 G: So, (moves hand from head and straightens posture), as the resistance increases it would take longer, wait, wait, time constant is the amount of time it takes to go to $1 / e$ of its original charge.

37 I: Okay.

38 G: Okay. So if it was a bigger resistance it would make sense that it would take longer, I think.

Gabi starts by describing the relationship between a large resistance and a longer discharge time. Though they state this qualitative relationship as a fact that "would make sense" (turn 38), we do not know why this makes sense to Gabi. Their reasoning could be related to a physical model of resistance blocking the flow of charges in a circuit and/or Ohm's law, which embodies this qualitative relationship mathematically. Although we cannot determine whether their warrant for why this makes sense is physical, mathematical, or something else, we see here that Gabi's "intuitive" reasoning is qualitative. Gabi also states the meaning of the time constant ("the amount of time it takes to go to 1/e of its original charge"), so they have laid out the pieces needed to conclude that $\tau \sim R$, though they do not explicitly state it here.

Next, Gabi moves onto thinking about the effect of capacitance on the discharge time:

38 G: And then if it was more capacitance, pshh, I have a hard time getting an intuitive grasp on like what capacitance is like (replaces hand on face), Like, so, 
capacitance is is charge over, per voltage (writes ' $Q / V$ '), I believe.

39 I: Mhm.

40 G: So that's like amount of charge that the capacitor can hold, at the, or ( $4 \mathrm{~s}$ pause). So like a capacitor (moves hand from face, placing hands parallel, separated by small distance) with larger capacitance ( $3 \mathrm{~s}$ pause), at the same like like charge, oh here we go (points to prompt), charged by the same amount, has more charge on it.

41 I: Okay.

42 G: So it makes sense if it has more charge on it, it would take longer, maybe sh, maybe longer to uh to uh discharge. Sure, we'll say okay, we'll say the time constant is $R C$.

During this section, Gabi's qualitative reasoning about capacitance takes two parts. First, Gabi states that a larger capacitance would have more charge (if all capacitors are "charged by the same amount," as Gabi says, presumably paraphrasing the situation given in the prompt, in which all capacitors are connected to the same $9 \mathrm{~V}$ battery). This is connected to the mathematical definition of capacitance, $C$ $=Q / V$, and Gabi's ratio interpretation that capacitance indicates the amount of charge the capacitor can hold at a certain voltage. (turn 38). Then, Gabi makes the qualitative conclusion that more charge would mean a longer discharge time, though again there is no evidence suggesting whether their warrant for this claim is physical, mathematical, or something else. As before, this lays out the pieces to conclude that $\tau \sim C$, though Gabi only indicates this and the previous potential conclusion that $\tau \sim R$ when stating that the time constant expression is $R C$ (turn 42).

In addition to helping students reconstruct equations, we propose that coherence between different ideas can also help students gain confidence in the face of uncertainty. Throughout this episode, Gabi expresses uncertainty not only about whether the time constant is $R C$ or $1 / R C$, but also about their reasoning throughout. Their explanations contain incomplete sentences and interruptions-for instance, "wait wait" (turn 36), "uh" (turn 42), "like" (turns 32, 38 and 40) or long run-on sentences punctuated by pauses (turns 32 and 40). Gabi's speech also includes hedges - for instance, "I think" (turns 32 and 38), "I believe" (turn 38), and "maybe" (turn 42), and explicit statements of uncertainty (turn 38: "I have a hard time getting an intuitive grasp on what capacitance is like."). In light of this uncertainty, coherence between different ideas - the mathematical expression for the time constant RC with "intuitive" qualitative relations between resistance, capacitance, charge, and discharge time - may provide the safety net to help Gabi believe in the conclusion that $\mathrm{RC}$ is the time constant. This may help explain why, after laying out an argument for $\tau \sim R$, Gabi reasons about capacitance even though knowing $\tau \sim R$ is sufficient to determine that $R C$ is plausible, and $1 / R C$ is not. Gabi's effort to understand capacitance intuitively here aligns with theories of coherence that argue that explanations with a greater number of connections between ideas are more preferable [16].

\section{B. Sam switches to another approach that doesn't require equations}

We also observe a community college student, Sam, noting that they forgot a relevant equation:

23 Sam: So I'm thinking um it's gonna discharge, and I forgot the formula again but it's cool, it's gonna discharge based on, you know, kind of like, it's like, I treat electrons kind of like people, which is weird, but whatever. I mean,

24 Interviewer: How do you mean?

25 S: like, they're, they kinda just make choices, you know.

26 I: Mhm. Mhm.

27 S: They kind of just pick their best fit scenario.

28 I: Mhm.

Sam was unable to remember a relevant formula, but they do not appear to be distressed by this. They switch from attempting to recall a mathematical expression to expressing an analogy of electrons as people. Sam then reasons physically about the circuit configuration to compare discharge rates:

29 S: So in this case, in circuit B (points to circuit B), they have, like, two paths, well, kind of. Yeah. They have like, those are kind of in parallel?

30 I: Mhm, mhm. They are in parallel. Yeah, yeah.

31 S: Yeah. They are. Yeah. So, they have two different paths, which means that, that that, well I wanna say that maybe the capacitor will discharge more.

32 I: Okay.

33 S: 'Cause it has two different ways to go.

34 I: And so, because, so two different ways to go for the current means, or for the electron.

$35 \mathrm{~S}$ : Mhm. Would mean that the, it would, discharge more quickly.

36 I: Okay.

37 S: I think. Versus, um, this one (points to circuit A), it only has one way,

38 I: Mhm.

39 S: So I think this one (points to circuit B) would be... faster, than this one (points to circuit A)?

Using the physical configurations, Sam determines that increased paths in circuit B's parallel circuit means it would discharge more quickly than circuit A (turns 34-35).

Next, Sam reasons how the smaller plate distance in circuit D would impact how quickly the circuit discharges:

41 S: I think this one (points to D) might be the fastest, though. Because of that distance.

42 I: What, what is the impact here? 
43 S: I don't know (chuckles).

44 I: What do you think?

45 S: Well, I think that, the, the distance between them, like I said it, the smaller distance (holds hands parallel to each other) increases capacitance. But if, wait, so they're all charged, they have the maximal possible charge,

46 I: Yeah.

$47 \mathrm{~S}$ : (reading from question) "Which capacitor has the most amount of charge left on it?" Oh, so maybe this one (points to D) will have, I was ranking them on how quickly, so,

48 I: Mhm.

$49 \mathrm{~S}$ : the one that, is the most, quick, is the, least, amount of charge.

50 I: Mhm. Mhm. Makes sense. Makes sense.

51 S: So maybe I'll call that (points to B) four. And then I think that (points to A) might be three.

52 I: Mhm.

53 S: (writes something next to $C$ and D) So I think this one [D] has the most amount of charge. Because it started with the most amount of charge.

54 I: Mhm.

$55 \mathrm{~S}$ : And, if you look at these two (points to A and D] which are the same other than that (points to capacitors on circuit A and D),

56 I: Mhm.

57 S: This one (points to D), I think, definitely would have more,

58 I: Mhm.

$59 \mathrm{~S}$ : if they're open for the same amount of, or closed for the same amount of time, because, of their, like starting, they started at a different amount,

60 I: Mhm. Mhm.

$61 \mathrm{~S}$ : and they're identical in every other way.

In the following moments, Sam explains that $\mathrm{C}$ ends with more charge than A, because C's greater resistance slows down the discharge.

Sam used qualitative reasoning to correctly determine that circuit $B$ has the least amount of charge remaining and circuit $\mathrm{D}$ has the most. They justify this by reasoning about the discharge rates and the starting amount of charge in the circuits. For instance, in turn 51, Sam notes that B ends with less charge than A, building on the conclusion in turn 39 that B discharges more quickly than A. Also, in turn 53, Sam states that D ends with the most charge, because it starts with the most charge. Although it is not entirely clear how they reach the conclusion that $D$ ends with the most charge, it is plausibly connected to the increased capacitance in circuit D. In a previous part of the interview, Sam concludes that smaller capacitor plate distance increases the capacitance (referenced in turn 45) and that greater capacitance corresponds to greater charge. And, notably, this reasoning about capacitors stems from another physical analogy: that capacitance indicates the ability of a capacitor to act like a battery.
Although there are aspects of the solution here that are incomplete (e.g., no verbalized comparison of the slower discharge rate of circuit $\mathrm{C}$ against the greater initial charge of circuit D), this excerpt shows that Sam has ways to reason about the situation to make progress on the problemqualitative reasoning tied to physical analogies - even after noting they forgot a relevant equation.

\section{DISCUSSION}

The cases of Gabi and Sam illustrate two different ways that students can make progress when facing one potential roadblock, forgetting a relevant equation. Where Gabi uses a coherence-seeking approach to decide between two possible forms of the RC time constant equation, Sam instead opts to reason qualitatively, in line with their physical understanding of circuits - an example that contributes to research on how students navigate qualitative and quantitative solution methods on physics problems [12].

These two cases illustrate ways that students' understanding of the physical situation provide corrective and alternative reasoning pathways when answering a physics problem. Specifically, they both relied on the ideas that the discharge time increases as resistance increases and as the starting charge on the capacitor increases. Gabi used these ideas to decide on the form of the time constant expression, while Sam used these ideas to compare the circuits qualitatively to answer the problem.

Gabi's coherence-based approach to reconstruct the equation adds another empirical example to a body of research showing that coherence between mathematics and physical understanding is a productive tool in physics reasoning and problem solving. There is some evidence that explicit efforts to emphasize this coherence in physics instruction can enhance students' problem-solving expertise [12]. Continuing to grow our understanding of the ways in which coherence between mathematics and physical understanding can be leveraged in physics problem solving may inform future instructional efforts.

This paper is an initial report out of a larger research project investigating how students coordinate and switch between multiple lines of reasoning during problem solving. Future directions for this project include further documenting the range of strategies students employ when forgetting an equation and exploring how students' epistemological stances towards problem solving can play a role in whether they switch to alternative strategies.

\section{ACKNOWLEDGMENTS}

This material is based upon work supported by the National Science Foundation and Department of Defense under Grants PHY-1659598 and PHY-1950744. Any opinions, findings, and conclusions or recommendations expressed in this material are those of the author(s) and do not necessarily reflect the views of the National Science Foundation or Department of Defense. 
[1] T. L. McCaskey, Comparing and Contrasting Different Methods for Probing Student Epistemology and Epistemological Development in Introductory Physics, PhD Thesis, University of Maryland (2009).

[2] P. Heller, R. Keith, and S. Anderson, Teaching Problem Solving through Cooperative Grouping. Part 1: Group versus Individual Problem Solving, American Journal of Physics 60, 627 (1992).

[3] J. I. Heller and F. Reif, Prescribing Effective Human Problem-Solving Processes: Problem Description in Physics, Cognition and Instruction 1, 177 (1984).

[4] J. L. Docktor, N. E. Strand, J. P. Mestre, and B. H. Ross, Conceptual Problem Solving in High School Physics, Physical Review Special Topics-Physics Education Research 11, 020106 (2015).

[5] B. R. Wilcox, M. D. Caballero, D. A. Rehn, and S. J. Pollock, Analytic Framework for Students' Use of Mathematics in Upper-Division Physics, Physical Review Special Topics-Physics Education Research 9, 020119 (2013).

[6] E. F. Redish and K. A. Smith, Looking beyond Content: Skill Development for Engineers, Journal of Engineering Education 97, 295 (2008).

[7] M. Park, Students' problem-solving strategies in qualitative physics questions in a simulation-based formative assessment, Discip. Interdiscip. Sci. Educ. Res. 2, 1 (2020).

[8] B. L. Sherin, How Students Understand Physics Equations, Cognition and Instruction 19, 479 (2001).

[9] A. Gupta and A. Elby, Beyond Epistemological Deficits: Dynamic Explanations of Engineering Students' Difficulties with Mathematical Sense-Making, International Journal of Science Education 33, 2463 (2011).

[10] S. Kapon and M. Schvartzer, Nurturing Sensemaking of, through, and with a Mathematical Model, in 2018 Physics Education Research Conference Proceedings (Washington, D.C., 2018).

[11] B. W. Dreyfus, A. Elby, A. Gupta, and E. R. Sohr, Mathematical Sense-Making in Quantum Mechanics: An Initial Peek, Physical Review Physics Education Research 13, 020141 (2017).

[12] E. Kuo, M. M. Hull, A. Elby, and A. Gupta, Assessing Mathematical Sensemaking in Physics through Calculation-Concept Crossover, Phys. Rev. Phys. Educ. Res. 16, 020109 (2020).

[13] J. D. Gifford and N. D. Finkelstein, Categorical Framework for Mathematical Sense Making in Physics, Physical Review Physics Education Research 16, 020121 (2020).

[14] R. S. Russ, V. R. Lee, and B. L. Sherin, Framing in Cognitive Clinical Interviews about Intuitive Science Knowledge: Dynamic Student Understandings of the Discourse Interaction, Science Education 96, 573 (2012).

[15] A. A. diSessa, An Interactional Analysis of Clinical Interviewing, Cognition and Instruction 25, 523 (2007).

[16] P. Thagard, Explanatory Coherence, Behavioral and Brain Sciences 12, 435 (1989). 